\title{
Experimental and Numerical Study of the Time-Dependent Pressure Response of a Shock Wave Oscillating in a Nozzle
}

\author{
P. OTT, A. BÖLCS, T. H. FRANSSON* \\ Laboratoire de Thermique Appliquée et de Turbomachines (LTT) \\ Swiss Federal Institute of Technology \\ Lausanne, Switzerland
}

\begin{abstract}
Investigations of flutter in transonic turbine cascades have shown that the movement of unsteady normal shocks has an important effect on the excitation of blades. In order to predict this phenomenon correctly, detailed studies concerning the response of unsteady blade pressures versus different parameters of an oscillating shock wave should be performed, if possible isolated from other flow effects in cascades. In the present investigation the correlation between an oscillating normal shock wave and the response of wall mounted time-dependent pressure transducers was studied experimentally in a nozzle with fluctuating back pressure. Excitation frequencies between $0 \mathrm{~Hz}$ and $180 \mathrm{~Hz}$ were investigated.
\end{abstract}

For the measurements, various measuring techniques were employed. The determination of the unsteady shock position was made by a line scan camera using the Schlieren flow visualization technique. This allowed the simultaneous use of unsteady pressure transducers to evaluate the behavior of the pressure under the moving shock.

A numerical code, based on the fully unsteady Euler equations in conservative form, was developed to simulate the behavior of the shock and the pressures.

The main results of this work were:

- The boundary layer over an unsteady pressure transducer has a quasi-steady behavior with respect to the phase lag. The pressure amplitude depends on the frequency of the back pressure.

- For the geometry investigated the shock amplitude decreased with increasing excitation frequency.

- The pressure transducer sensed the arriving shock before the shock had reached the position of the pressure transducer.

- The computed unsteady phenomena agree well with the results of the measurements.

\section{INTRODUCTION}

Unsteady flow effects in turbomachines can be the source of various excitations that can lead to different vibrations, such as forced vibration and flutter. Turbomachine blade vibrations are known to appear over a large flight envelope for compressors in jet engines and on the last stages of industrial turbines. Main vibration problems appear in the transonic flow domain (Bölcs et al, 1989b; Ezzat et al, 1989; Usab and Verdon, 1990; Verdon, 1989; Araki et al, 1981; Széchényi et al, 1984, 1985; Buffum and Fleeter 1989, 1990, 1991; Hanamura, 1988; Fransson, 1992 to mention just a few) and it has been noted both theoretically and experimentally that oscillating normal shock waves can introduce large unsteady local loads on the vibrating blades [Verdon, 1989; Bölcs et al, 1991]. Because of the small blade dimensions usually employed for cascade experiments in the transonic flow domain it is extremely difficult to obtain accurate information about the exact position of the shock wave, how much it fluctuates, its harmonic and non-harmonic content, as well as the correlation between the "freestream" (outside the boundary layer) shock position and the time-dependent pressure responses on the blades.

Numerical predictions are presently performed for both the forced vibration and the flutter problem. In these, emphasis is sometimes put on the sharp shock capture [Carstens, 1991] or shock fitting [Verdon, 1989], whereas other results indicate that less sharp shock capture gives smaller local unsteady loads but over a more spread out region [Whitehead et al, 1985, 1990; Gerolymos, 1992a,b], and that the total chordwise load agrees with global experimental data.

For the correct interpretation of both experimental data and numerical predictions of the unsteady transonic flow around vibrating cascaded airfoils it is important to determine the correlation between the:

\footnotetext{
presently: Division of Heat and Power Technology, Royal Institute of Technology, Stockholm, Sweden
} 
- oscillating shock wave and fluctuating blade pressures

- experimental and numerical results.

Previous numerical two-dimensional investigations in a nozzle [Bölcs et al, 1989a, Liang et al, 1992] indicated that both the amplitude and frequency of an imposed fluctuating back pressure are important parameters for the location of the unsteady shock. This study also indicated that the pressure jump over the shock, as well as the unsteady post-shock pressure, is different for identical shock positions during the cycle of fluctuation.

Similar measurements as here presented were made by Edwards [1987]. The conditions were slightly different. The flow investigated was on a flat surface with a flow separation behind the shock. The boundary layers were thinner and especially the resolution of the device to find the shock position was smaller. This and some supplementary questions to answer made it necessary to re-investigate the problem.

The objectives of the present work are to obtain fundamental data for the correlation between an oscillating shock wave and the time-dependent pressures measured on a nozzle wall, in the presence of a thick boundary layer, further to validate an improved version of the numerical model previously presented against experimental data on oscillating shock waves in a nozzle.

\section{NOMENCLATURE}

a

$\vec{F}$

$\mathrm{D}$

$e_{c}$

f

$\vec{G}$

$\mathrm{h}_{\mathrm{c}}$

$M$

$\mathrm{p}$

$\tilde{p}$

ptot

q

$\mathrm{R}$

$\mathrm{Re}$

$\mathrm{t}$

$T$

U

V

$\vec{W}$

$\mathrm{x}$

$y$

$\eta$

$\kappa$

$\xi$

p

$\tau$

$\omega$ velocity of sound

flux vector

Jacobian determinant

total energy/unit mass

frequency

flux vector

total enthalpy/unit mass

Mach number

pressure

pressure (unsteady perturbation)

total pressure

velocity

gas constant ( $\approx 287$ for air)

Reynolds number

time

temperature

velocity component in $\mathrm{x}$-direction

velocity component in $\mathrm{y}$-direction

flux vector

coordinate direction

coordinate direction

ratio of specific heats

coordinate direction

density

time

circular frequency coordinate direction $\mathrm{m}^{2} /\left(\mathrm{s}^{2} \mathrm{~K}\right)$ mbar, $\mathrm{m} / \mathrm{s},-$

$$
\text { s, - }
$$$$
\mathrm{K},-
$$

$\mathrm{m} / \mathrm{s},-$

$\mathrm{m} / \mathrm{s}$, -

$\mathrm{mm}$, -
$\mathrm{mm}$, -

\section{Subscripts and Superscripts}

1 inlet condition

c stagnation value

ref reference value

$\mathrm{n} \quad$ pressure transducer number $(\mathrm{n}=1 \ldots 8)$

$x \quad$ in $x$-direction

$y \quad$ in $y$-direction

$\xi \quad$ in $\xi$-direction

$\eta \quad$ in $\eta$-direction

\section{MEASURING EQUIPMENT}

\section{Test Facility}

A Laval nozzle with a width of $40 \mathrm{~mm}$ was modified for the purpose of the present study. The facility was equipped with nozzle liners giving a convergingdiverging section with a continuous first derivative of the area (Fig. 1 and Appendix 1). The inlet and outlet height of the test section is $80 \mathrm{~mm}$ and the throat height is $71 \mathrm{~mm}$. A window is situated in the aft part of the convergent-divergent section and gives access to nonintrusive measurement techniques.

A four-stage radial, continuously running turbocompressor (pressure ratio $4,10 \mathrm{~kg} / \mathrm{s}$ mass flow) is used as air source.

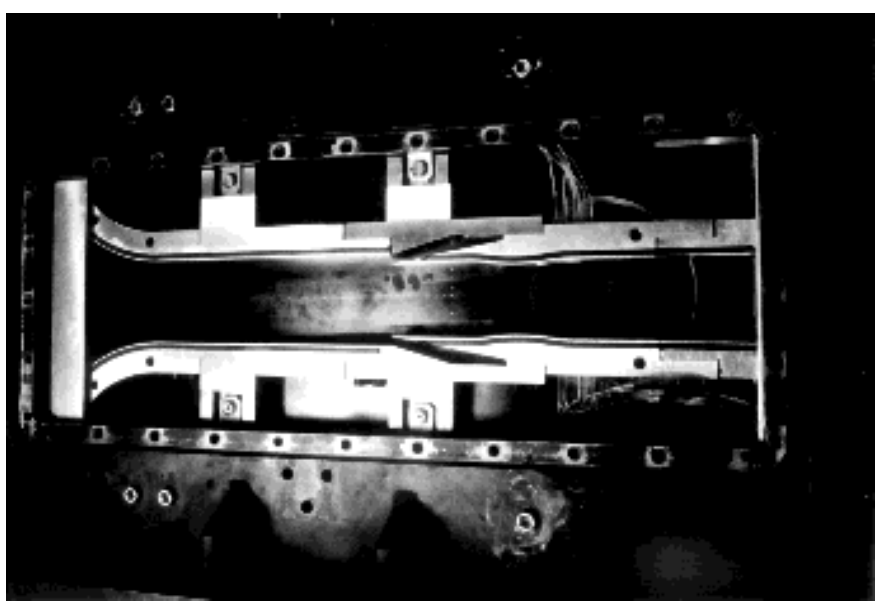

Fig. 1a: Photo of the nozzle

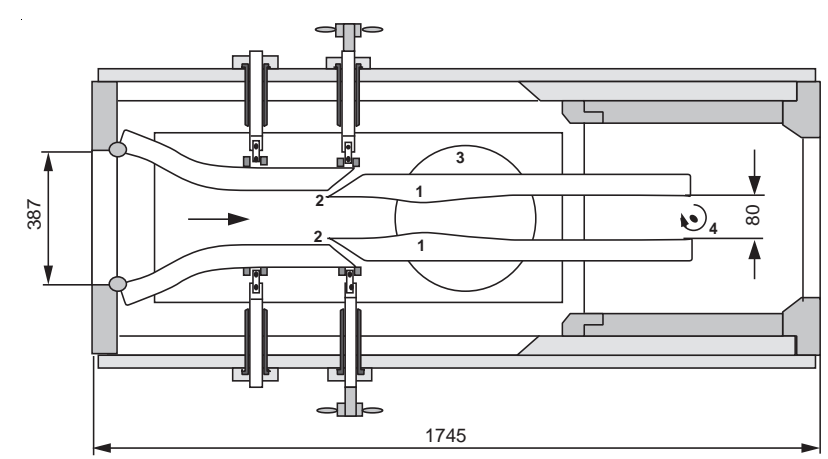

$\mathrm{kg} / \mathrm{m}^{3}$, - 1 nozzle liner 3 Schlieren window 2 boundary layer bleed 4 rotating exciter $\mathrm{rad} / \mathrm{s} \quad$ Fig. 1b: Schematic view of the nozzle used 
On the nozzle liners the boundary layer is cut off before the throat (Fig. 1). No suction of this mass flow is performed, and the air is blown by the flow behind the nozzle liners and again mixed with the main stream at the exit of the nozzle. The boundary layers at the side wall are not modified, which creates the fairly thick boundary layer used in the study.

\section{Oscillating shock}

A normal shock is created in the test section of the nozzle by regulating the exit pressure of the nozzle.

At the exit section of the nozzle formed by the liners a cylindrical rod with elliptical cross section (Fig. 1b) is rotated by a hydraulic motor. This rod is situated $480 \mathrm{~mm}$ downstream of the throat. The losses created by the wake of the rod are varied in time and in this way the outlet pressure of the nozzle is changed periodically. By this variation the normal shock is excited to oscillation frequencies between 0 and $180 \mathrm{~Hz}$. For the present tests the shock is always oscillating in the divergent section of the nozzle. Upstream of the shock, depending on its position, Mach numbers between 1.2 and 1.4 are reached.

\section{Instrumentation}

On the nozzle liners and on the side walls steady state pressure taps exist and give the steady state pressure distribution through the nozzle.

For pressure measurements the mirror plate of the double-pass Schlieren system (" 3 " in Fig. 1b) is equipped with 80 pressure taps where either small tubes for static pressure measurements or tubes containing a high frequency response pressure transducer $^{1}$ can be screwed in. Eight tubes containing pressure transducers were used for the present test series. The mounting was performed in a way that the pressure transducers were sitting just behind the surface of the mirror plate with a dead volume that is negligible. The transducers were calibrated for steady state and dynamic pressure, including the whole measuring chain (dead volume, cables, amplifiers, filters, etc.), while they were mounted in their corresponding measuring positions.

The shock form and position is observed by a reflecting Schlieren system using a video camera and a stroboscopic flash synchronized with this camera. Furthermore, the shock position, its form, the boundary layers and the flow velocities up- and downstream of the shock, are also determined with a Laser-2-Focus system, both for "steady-state" and time-dependent operating conditions at different frequencies. Finally, holographic interferometry was used to get some supplementary information about the boundary layers on the nozzle liners close to the shock for the steady state flow.

1 Pressure transducer ENDEVCO, model PS 8514-50, range: 0 - 3.4 bar, accuracy: $0.5 \%$ Full Scale

For the time-dependent measurements the flash is replaced by a continuous light source. The shock position is found by a line scan camera ${ }^{2}$ which observes one line of the Schlieren picture normal to the shock (Fig. 2). A mask with well-known position is fixed on the window for determination of the geometric location of the shock in the channel. The distance left free by the mask was $40 \mathrm{~mm}$ for these tests. The Schlieren image is projected on the sensitive line of the camera composed by 1024 photodiods (pixels). Under consideration of about 800 pixels within the range of the distance left free by the mask, a spatial resolution of $0.05 \mathrm{~mm}$ is reached. Fig. 3a shows a schematic view of the flow field and a theoretical resulting signal of the line scan camera. Fig. 3b shows a real signal. The so found shock position is integrated over the nozzle height. Simultaneously to the camera signal the unsteady pressure distribution is measured by the timedependent pressure transducers fixed in the mirror plate at the side wall.

The signals of the camera and the pressure transducers are recorded, together with a triggering signal of the exciter, on an analog data recorder ${ }^{3}$ and digitized off-line. The digitized signals of the line scan camera can give the position of the shock in the moment when the line scan camera "froze" the instantaneous picture before writing out the voltage of all diodes (Fig. 3b).

After elimination of the time shift between the different signals due to the multiplexer used during the digitalization, a signal averaging for one excitation period is made for the pressure signals and the shock positions. The results can be presented in a form as shown by Fig. 7 .

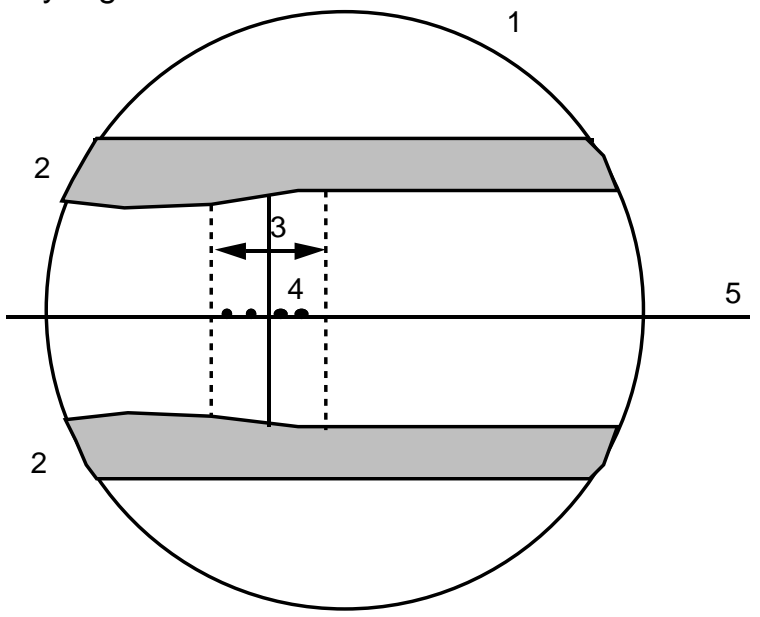

1 Schlieren window

2 nozzle liner

3 moving shock

\section{scan camera}

Fig. 2: Position of observed line and pressure transducers
2 Line scan camera Reticon, model LC-1901

3 Tape recorder KYOWA, model RTP-802A 


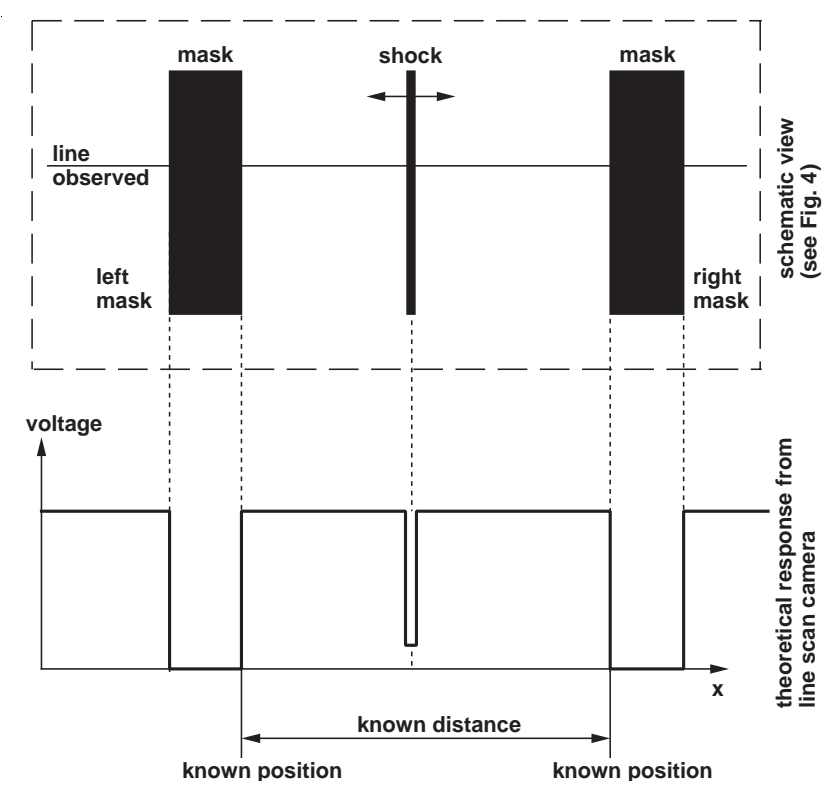

Fig. 3a: Schematic illustration of Schlieren visualization and a line scan camera signal

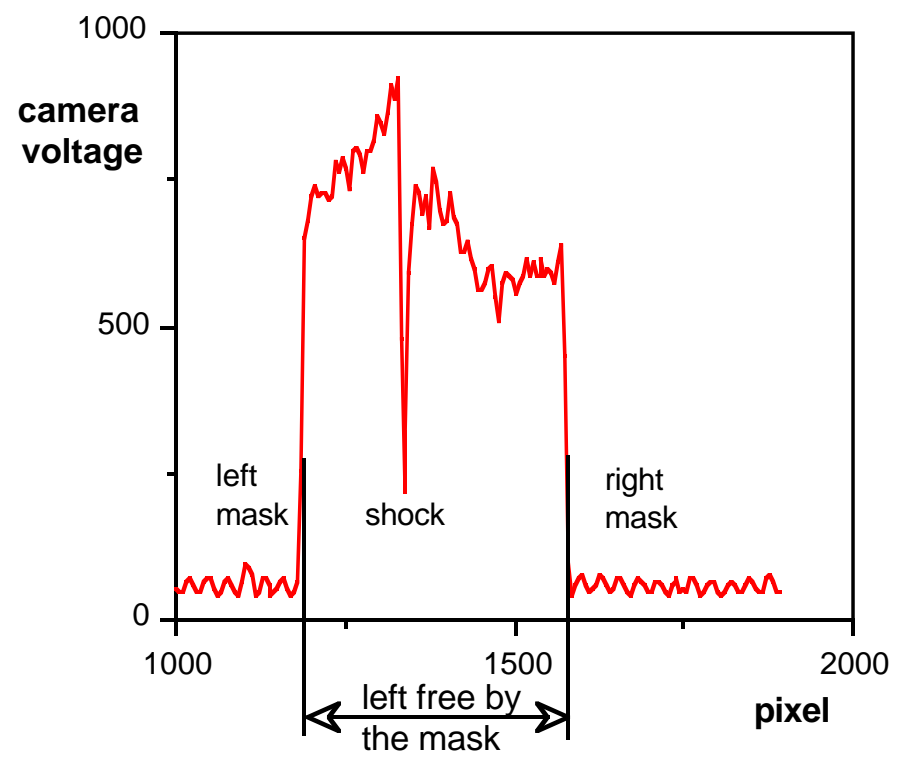

Fig. 3b: Untreated signal of line scan camera with shock

The trigger signal allows to locate the moment when the picture was taken inside one excitation period. As the phenomenon studied is periodic a signal averaging for the pressure transducer signals and for the shock positions can be made. All results are then presented within one excitation period.

Fig. 4 shows the positions of the unsteady pressure transducers for the measurements here presented. The pressure transducers were positioned such that some are located slightly outside of the shock oscillation range, and others where the shock always would be present. One transducer was mounted as far downstream from the shock as possible to measure the pressure oscillation at the outlet (pressure transducer $\mathrm{p}_{8}$ in Fig. 4).

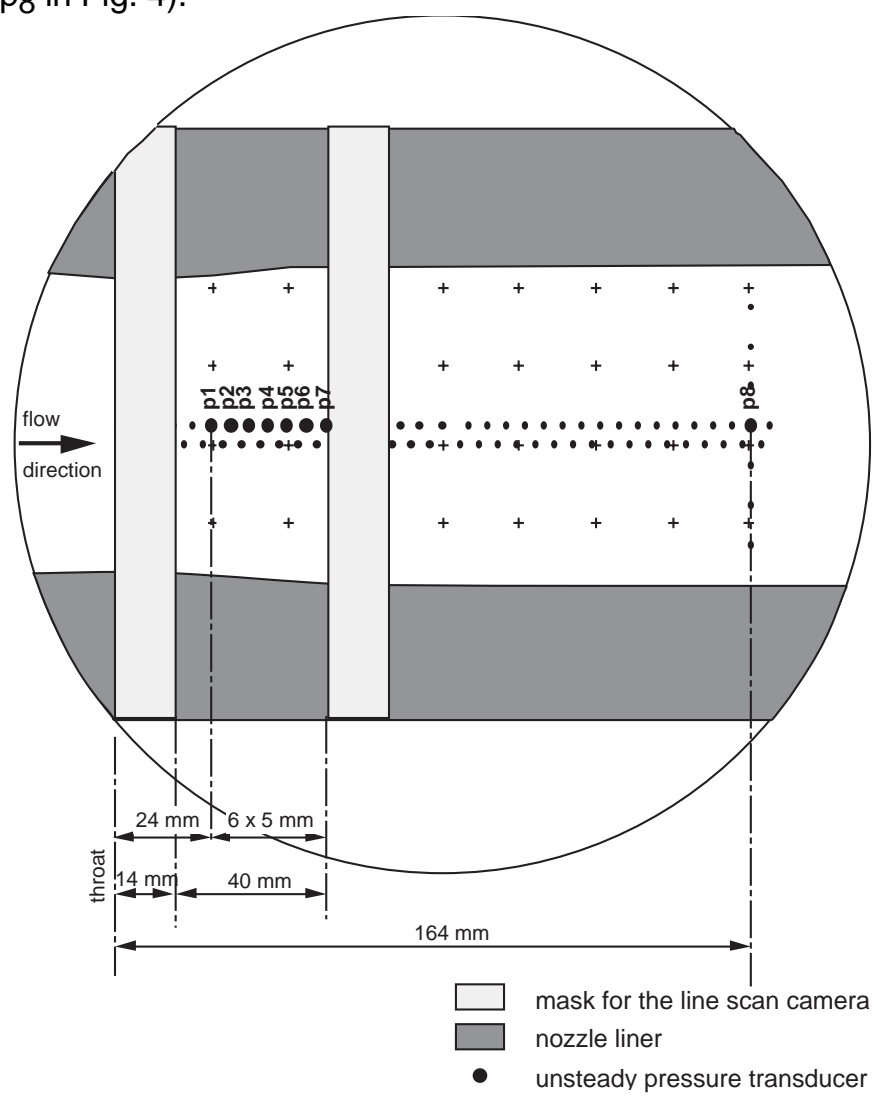

Fig. 4: Positions of the unsteady pressure transducers during the tests

More details of the measuring equipment have been given by Ott et al [1992].

\section{GOVERNING EQUATIONS AND NUMERICAL METHOD OF SOLUTION}

\section{Governing Equations}

The computational method used here is an explicit, inviscid two-dimensional model of second order accuracy, based on the Euler equations in conservative form. The model has been extended from a previous work [Bölcs et al, 1989a], and only a brief explanation of the model is thus given here.

The equations of continuity, momentum and energy, which form the basis for the formulation, can be expressed in vector form:

$$
\vec{W}_{t}+\vec{F}_{x}+\vec{G}_{y}=0
$$

The vectors can be expressed as:

$$
\vec{W}=\left(\begin{array}{c}
\rho \\
\rho u \\
\rho v \\
\rho e_{c}
\end{array}\right) ; \vec{F}=\left(\begin{array}{c}
\rho u \\
\rho u^{2}+p \\
\rho u v \\
\rho u h_{c}
\end{array}\right) ; \vec{G}=\left(\begin{array}{c}
\rho v \\
\rho u v \\
\rho v^{2}+p \\
\rho v h_{c}
\end{array}\right)
$$


The equation of state for a perfect gas is used to find the relations between pressure, density and temperature.

All variables presented in these equations have been made dimensionless by use of the following reference values $p_{\text {ref }}, x_{\text {ref }}=y_{\text {ref }}, T_{\text {ref }}$, as well as:

$$
\begin{array}{ll}
\rho_{\text {ref }}=\frac{p_{\text {ref }}}{R \cdot T_{\text {ref }}} & q_{\text {ref }}=\sqrt{R \cdot T_{\text {ref }}}=a / \sqrt{\kappa} \\
t_{\text {ref }}=\frac{x_{\text {ref }}}{q_{\text {ref }}} & e_{\text {ref }}=h_{\text {ref }}=q_{\text {ref }}^{2}
\end{array}
$$

The equation system (1) is mapped from the physical plane $(x, y, t)$ to the computational plane $(\xi, \eta, \tau)$ with a general transformation:

$$
\begin{aligned}
& \xi=\xi(x, y, t) \\
& \eta=\eta(x, y, t) \\
& \tau=t
\end{aligned}
$$

Equation (1) becomes after this transformation:

$$
\vec{W}_{t}^{\prime}+{\overrightarrow{F^{\prime}}}_{\xi}+\vec{G}_{\eta}=0
$$

with the vectors:

$$
\begin{aligned}
& \vec{W}^{\prime}=D^{-1} \cdot \vec{W} \\
& \overrightarrow{F^{\prime}}=D^{-1} \cdot\left\{\xi_{t} \cdot \vec{W}+\xi_{x} \cdot \vec{F}+\xi_{y} \cdot \vec{G}\right\} \\
& \overrightarrow{G^{\prime}}=D^{-1} \cdot\left\{\eta_{t} \cdot \vec{W}+\eta_{x} \cdot \vec{F}+\eta_{y} \cdot \vec{G}\right\}
\end{aligned}
$$

where $D$ is the Jacobian determinant of the transformation (5) in the center of a computational cell and corresponds physically to the inverse of the cell area.

The solution follows with an upwinding model, in which the flux-vectors are split in positive and negative fluxes.:

$$
\begin{aligned}
& \vec{F}=\vec{F}++\vec{F}- \\
& \vec{G}=\vec{G}++\vec{G}-
\end{aligned}
$$

The positive and negative fluxes are determined with respect to the physical domain of influence by the fluxvector splitting method of van Leer [Anderson et al, 1987]. For a supersonic flow in x-direction, for example, the negative part of the flux becomes zero; all flow information comes from upstream.

\section{Numerical Grid}

For the computations here presented a $\mathrm{H}$-mesh was used with 81 mesh points in stream-wise direction $(x)$ and 15 mesh points normal to this direction $(y)$. The first row of points is situated half a mesh distance from the walls of the nozzle.

\section{Spatial Differencing}

The numerical model uses the approach of "Monotone Upwind Schemes for Conservation Laws (MUSCL)" proposed by van Leer [Anderson et al, 1985]. In this approach the data are first prepared and eventually limited before the numerical differences are performed. The spatial derivatives are approximated as centered differences with values at half points.

A flux limiter is used at the location of the shock to reduce the accuracy of the computation to the first order.

\section{Integration in Time}

For the advance in time a two-step predictor-corrector method after MacCormack was used.

\section{Boundary conditions}

The boundary treatment used at the inlet for the computations here presented is the so called "capacitive boundary condition". It represents an inlet with constant total pressure, temperature and imposed flow direction. A perturbation impinging on this border is reflected back into the flow field. The flow variables on this border are determined by the method of characteristics.

The boundary treatment used at the outlet corresponds in its quality to that chosen for the inlet. Here, the static pressure is imposed as a function of time.

\section{RESULTS AND DISCUSSION \\ Steady State Flow}

Two different steady state flows were realized: with the exciter in its horizontal position the most downstream shock position is obtained, with the exciter in its vertical position the most upstream shock position is reached. In this way, the maximum and minimum outlet pressures are obtained for the inlet flow conditions under consideration here $\left(M_{1}=0.70\right.$, $p_{1}=1215$ mbar, $p_{\text {tot } 1}=1680$ mbar). The Reynolds number during these tests was $1.0 \cdot 10^{6}$, based on the inlet flow and the throat height.

Fig. 5 shows the steady state pressure distribution on the side wall of the nozzle for both extreme positions for measurement and computation. The pressure jump measured at the side wall is not as sharp as it is expected. The reason is the interaction between the shock and the boundary layer at the side wall. In the middle of the channel the pressure behaves much more similar to the inviscid computations. 


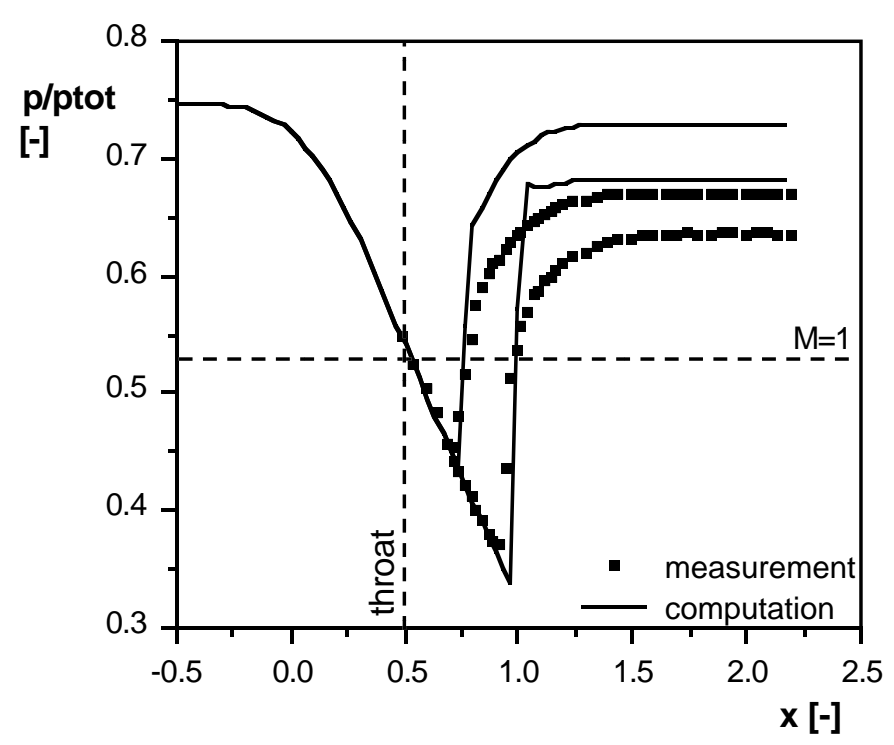

Fig. 5: Steady state pressure distribution (extreme values) on nozzle side wall together with numerical results (1 mesh size $\triangleq x=0.0333[-])$

By using the static pressures measured in the aft of the nozzle for both extreme positions of the shock as input for the computation it was observed that the shock positions found by the computation did not correspond with the shock positions measured. This was expected because of the presence of large boundary layers on the side walls. The losses due to the boundary layers mean an acceleration of the flow speed and a shock position more upstream than in an inviscid flow.

An inviscid computation can not reproduce the effects of these boundary layers. For the later discussion of unsteady results, a correlation was made with the program by changing the back pressure until the computed shock position corresponds with the measured one. This was made for both extreme shock positions.

An estimation of the increasing displacement thicknesses of the boundary layers after the shock measured with a L2F velocimeter shows that the order of magnitude for the acceleration of the flow speed due to the smaller section corresponds to the difference of static pressure found by the above mentioned iteration.

By different means, including Laser-2-Focus velocimeter and Pitot probes, large boundary layers on the side walls, up to $12 \mathrm{~mm}$ behind the shock, were observed. These large boundary layers lead to a flow information transport from the subsonic region downstream to the supersonic region upstream. The pressure transducers respond to the presence of the shock in a larger region than it is expected in a flow with smaller boundary layers.

\section{Unsteady Flow Effects}

Once the shock positions have been found for the two extreme "steady-state" operating conditions, the exciter is put into rotation. Fig. 6 shows the extreme values of the shock positions measured with the line scan camera for different excitation frequencies. It is clearly seen that the amplitude of the shock decreases with increasing frequency. The mean shock position remains, for the present geometrical and flow configuration, in the same location. The same observations can be made for the numerical results which are presented in the same diagram.

The exit pressure did not show a constant amplitude for all excitation frequencies during the measurements. The amplitude decreased with increasing excitation frequency. This is normal since there must be a limit condition at very high frequencies where the pressure will be stable and the shock will not move any longer. The pressure amplitude at the outlet for the computation as it was found by the above mentioned iteration for $0 \mathrm{~Hz}$ excitation frequency was thus changed in the same scale as it was measured by the pressure transducer $p_{8}$ (Fig. 4). Appendix 2 shows the mean exit pressure and the pressure amplitudes used for the unsteady computations here presented.

The results of the computations fit very well the extreme shock positions measured. The agreement is given by both mean shock position and shock amplitude.

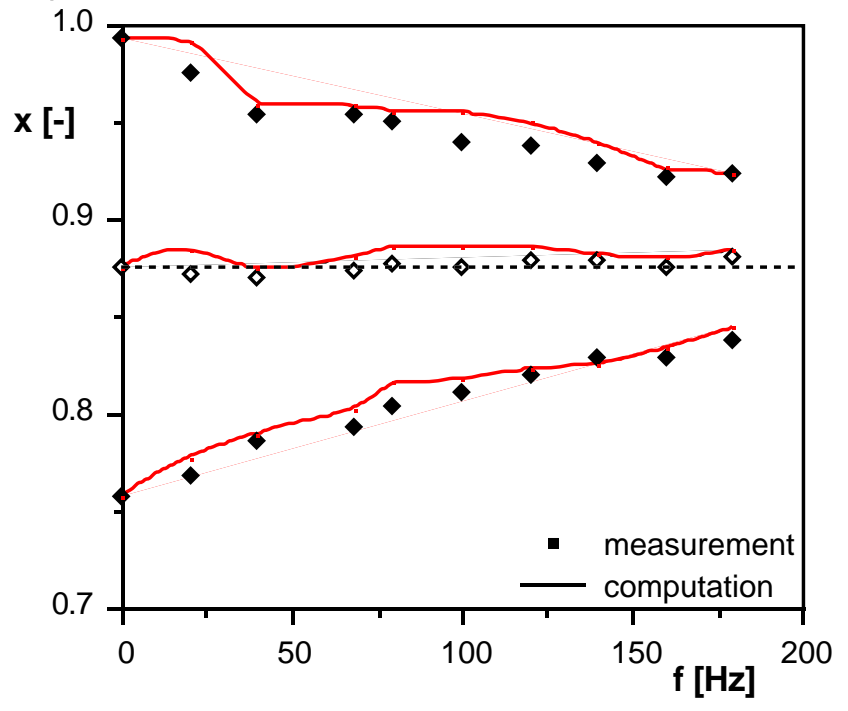

Fig. 6: Extreme shock positions for different excitation frequencies (measurement and numerical results)

The evolution of the unsteady pressures measured by the different transducers on the side wall together with the shock positions as determined by the line scan camera are shown in Fig. 7 for three different excitation frequencies $(f=20,80,180 \mathrm{~Hz})$. All results are presented within one excitation period. The positions of the pressure transducers are marked in the diagram with the shock position, i.e. the lower diagram. The presentation chosen allows to correlate the signal of a pressure transducer with the motion of the shock wave with the aid of the vertical lines relating the upper and lower diagrams in each figure. This correlation shows 
clearly that the pressure transducers respond by a change in the pressure before the shock reaches the corresponding geometrical locations. For small excitation frequencies the upstream influence distance corresponds to expected steady state values [Inger, 1981]. For higher frequencies the upstream influence distance increases for a shock moving downstream while it remains in the same order of magnitude as for small frequencies for the shock moving upstream, as can be seen from Fig. 7. For the highest excitation frequencies examined there exists no longer a domain of constant pressure while the shock is far away from the location of the pressure transducer.

It is clearly noted that the average shock movement is harmonic, whereas the pressure transducer response obviously is non-harmonic for the pressure transducers which are directly under the influence of the moving shock. Nevertheless, it is also observed that the pressures have their minimum or maximum values at the same instant as the shock is in one of its extreme positions. The boundary layer increases the zone of influence of the shock, but there is no phase lag between the shock movement, as measured with the line scan camera and the Schlieren system, and the unsteady pressure evolution. This conclusion is valid for all frequencies up to $180 \mathrm{~Hz}$.

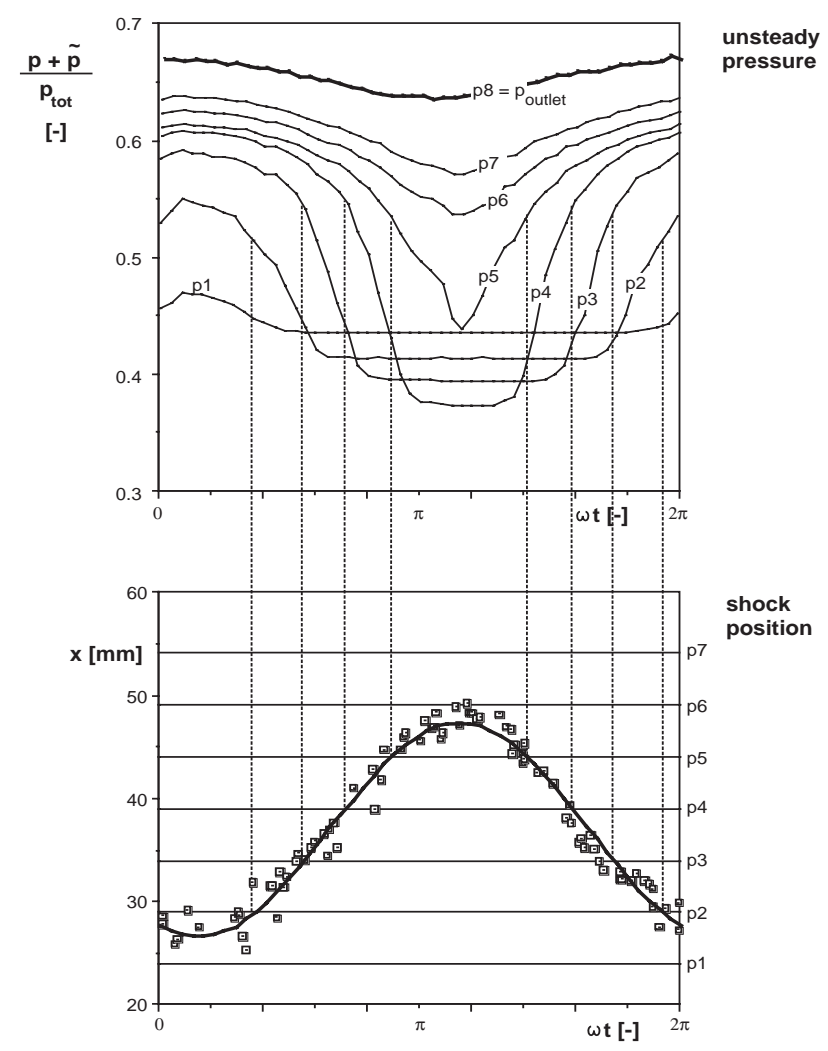

Fig. 7a: Unsteady pressure evolution together with shock position (excitation frequency $20 \mathrm{~Hz}$ )

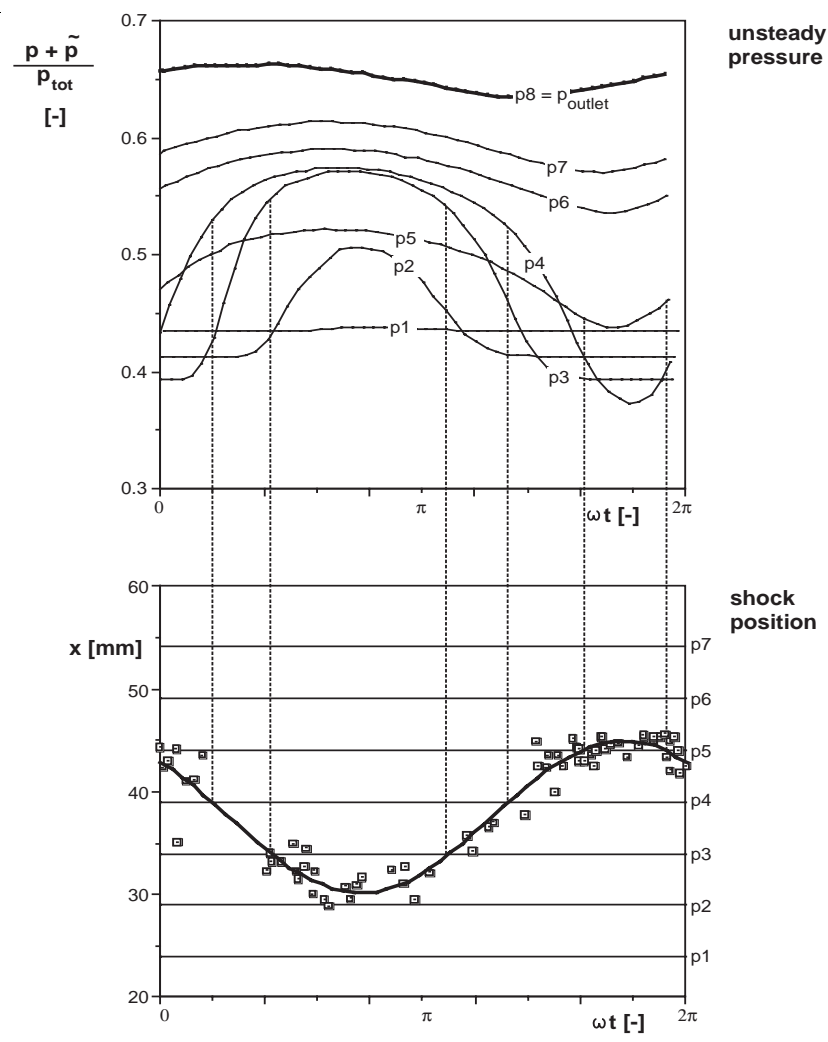

Fig. 7b: Unsteady pressure evolution together with shock position (excitation frequency $80 \mathrm{~Hz}$ )

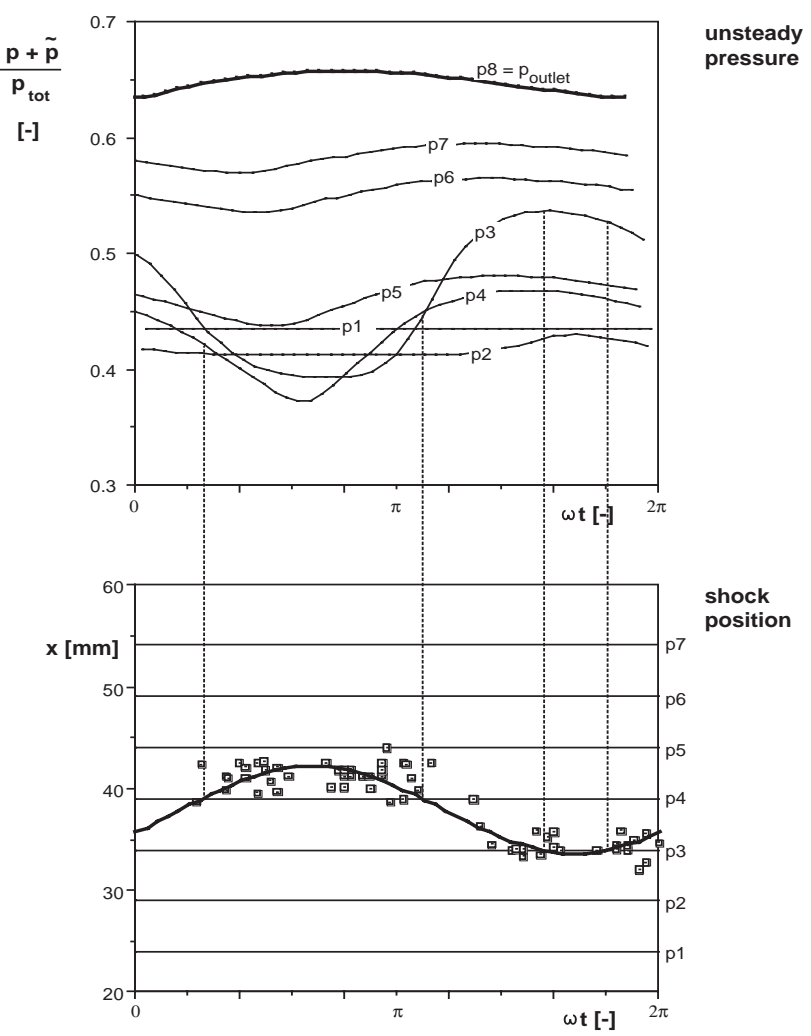

Fig. 7c: Unsteady pressure evolution together with shock position (excitation frequency $180 \mathrm{~Hz}$ ) 
It is seen from Fig. 7 that the pressure amplitude at a certain location decreases with increasing frequency. Fig. 8 shows the evolution of the pressure amplitude on the side wall for the different pressure transducer locations. The pressure transducer $p_{1}$ is never situated under the moving shock: For small excitation frequencies it feels a small pressure perturbation which comes through the boundary layer. For higher frequencies there is no signal at all, since this pressure transducer is always situated in the supersonic region and the distance to the extreme shock position is increasing with increasing frequency. The pressure transducers $p_{6}$ and $p_{7}$ are also never influenced directly by the shock. But since they are always located in the subsonic region, they show for high excitation frequencies, the same behavior as the pressure transducer $p_{8}$ which is situated far downstream of the shock; obviously with a certain phase lag corresponding to the time taken by the disturbances to travel from $p_{8}$ to $\mathrm{p}_{7}, \mathrm{p}_{6}$. The signals of pressure transducers which are situated for all excitation frequencies under the moving shock are more difficult to understand. The shock strength at this location is expected to be independent of the frequency. The flow speed before the shock is given by the shape of the nozzle. The shock velocity can be neglected compared to the flow velocity for the consideration of the strength of the shock. The reason for the diminution of the signal of the pressure transducers is the presence of the boundary layers. The pressure transducers measure at the side walls under a thick boundary layer of up to $12 \mathrm{~mm}$. In Fig. 9 the evolution of the pressure under the shock is shown for the middle of the channel and for the side wall. For an increased excitation frequency the pressure variation at the location of the pressure transducer at the side wall is smaller even if the pressure variation in the middle of the channel remains unchanged.

From the curves in Fig. 8 it is seen that the amplitude of pressure transducer $p_{4}$ decreases faster than the signal of pressure transducer $p_{3}$. In Fig. 6 and 9 it can be seen that the mean shock position is closer to $p_{4}$ than to $p_{3}$. The pressure transducers $p_{3}$ and $p_{4}$ should thus behave opposite to what is seen in Fig. 8. Their behavior becomes more comprehensive by looking at the shape of the shock over the channel width (Fig. 10). The shock positions marked so far are the positions in the middle of the channel. At the side walls the shock is situated more upstream than in the middle of the channel. The mean shock position at the side walls is closer to $p_{3}$ than to $p_{4}$. This is also indicated by the behavior of the pressure transducers.

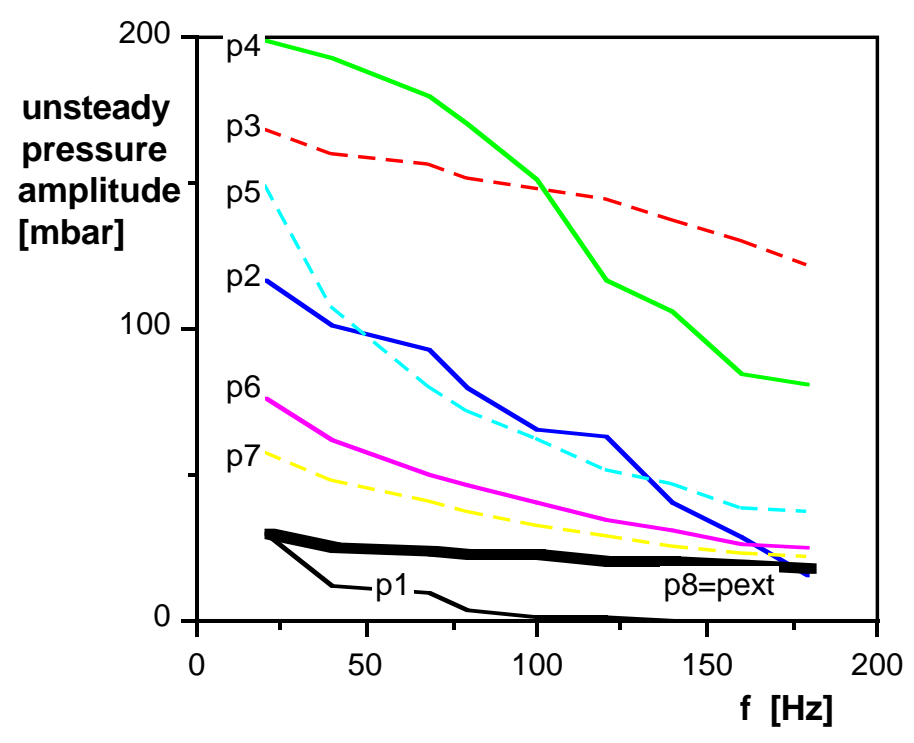

Fig. 8: Behavior of the pressure amplitude at the side wall for different excitation frequencies
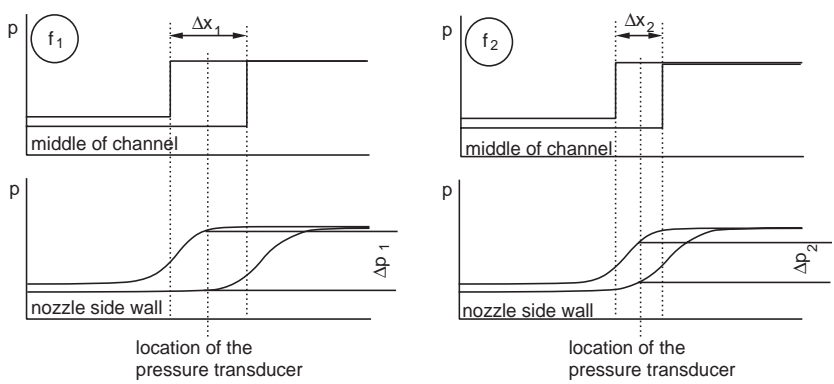

excitation frequency: shock displacement: static pressure amplitude:

$$
\begin{aligned}
\mathrm{f}_{1} & <\mathrm{f}_{2} \\
\Delta \mathrm{x}_{1} & >\Delta \mathrm{x}_{2} \\
\Delta \mathrm{p}_{1} & >\Delta \mathrm{p}_{2}
\end{aligned}
$$

Fig. 9: Schematic comparison of the static pressure evolution in the middle of the channel and at the side wall for two different excitation frequencies 


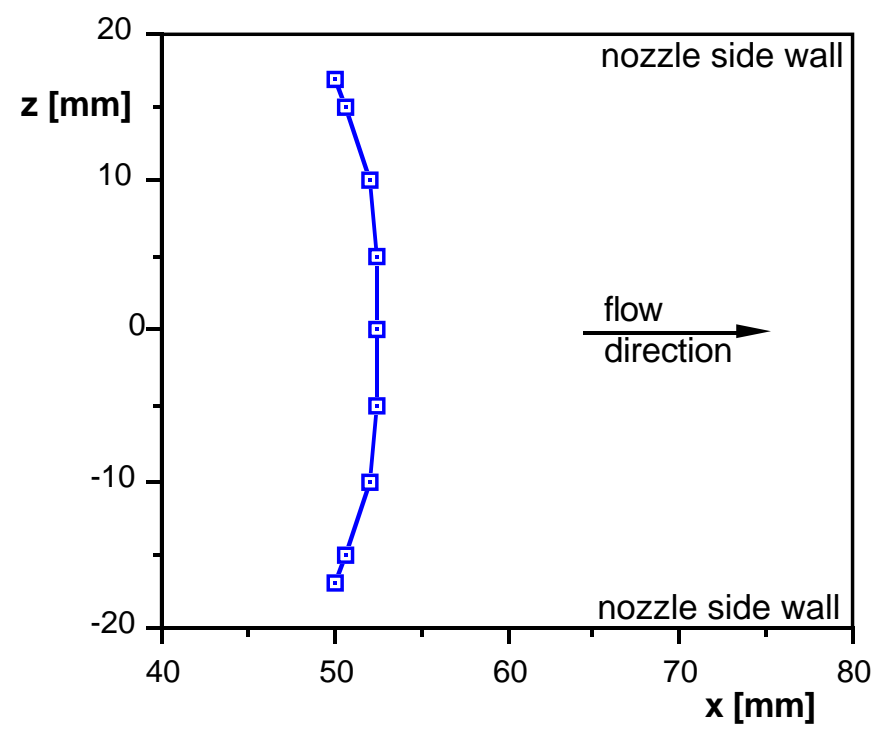

Fig. 10: Shock form over the channel width (measured with $L 2 F$ velocimeter)

Fig. 11 shows a comparison of the measured and the computed pressure distributions together with the shock position for the excitation frequencies of $80 \mathrm{~Hz}$. The shock position for the computation shows waviness in its evolution. The shock position is found by an interpolation algorithm outgoing from the flow variables at the different mesh points to find the location where the flow speed corresponds to the sonic velocity. The small ondulations that can be seen in the trace of the shock drawn in Fig. 11 probably appear because of an irregularity of the shock motion when the shock passes over a grid point. It can be seen, that the magnitude of the measured pressures is lower than the computed pressures. This is also due to the influence of the viscous flow effects which accelerate the flow and which can not be reproduced by the inviscid computation. The influence distance of the shock due to the presence of the boundary layers can clearly be seen by the comparison with the inviscid computations. The boundary layer transports the pressure information of the arriving shock in its subsonic part. In inviscid computations no boundary layer exists and what seems to be the influence distance corresponds simply to the distance between two points of the computational mesh. As soon as the shock passes the neighbor point the pressure begins to change. For the inviscid computations the influence distance seen in Fig. 11 has no physical meaning. The difference between measurement and computation shows the magnitude of the influence of the viscous flow effects.

Nevertheless both, measurement and computation, show the extreme values of the pressure evolution at the same moment as the shock reaches one of its extreme positions. There is no phase lag between shock movement and the pressure response.

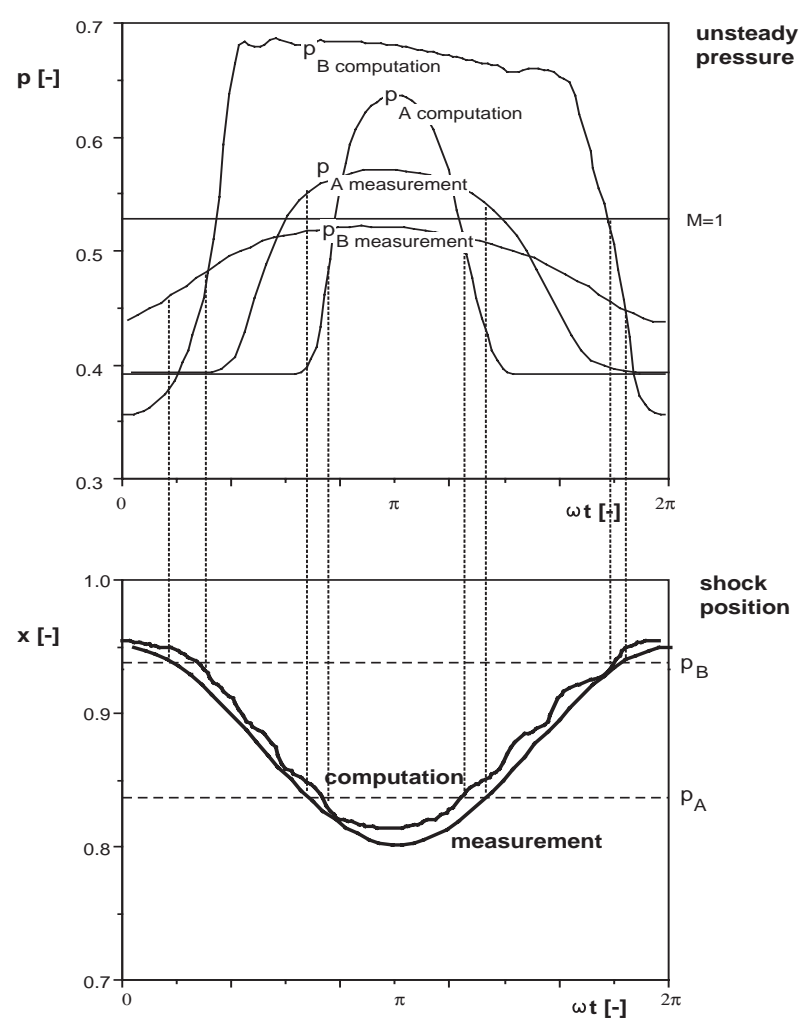

Fig. 11: Unsteady pressure evolution together with shock position (excitation frequency $80 \mathrm{~Hz}$ ) (measurement and computation)

Further information about the steady state and unsteady results can be found in Ott [1992].

\section{CONCLUSIONS}

Experimental and numerical investigations on the behavior of an oscillating normal shock and the effects of this shock to the response of unsteady pressure transducers have been executed. The observations and conclusions made during this study are supposed to help for the interpretation and judgment of measurements and observations made in oscillating turbine or compressor cascades in the transonic flow domain. The numerical studies should help for the development of a numerical model for unsteady cascade computations. The observations and conclusions are as follows:

- For the reduced frequencies studied here $(\mathrm{k}=0->0.18)$ it is permissible to consider the timedependent pressure response on a wall as quasisteady concerning the phase lag. No phase shift could be found between the oscillating shock wave and the side wall pressure response.

- The influence of the shock wave is noticed, due to the presence of the boundary layer, in the pressure response upstream of the shock position. This influence distance comports for low excitation frequencies as expected. For higher frequencies the upstream influence distance is larger for a shock 
moving forwards in streamwise direction than for a shock moving backwards.

- The amplitude of the shock oscillation decreases with increasing excitation frequency.

- The evolution of the unsteady pressure at the side wall under the normal shock passing the location of the transducer has a big non-harmonic behavior.

- Even if the viscous effects are important, an inviscid computational method can correctly reproduce the shock position and the shock amplitude.

- The comparison between measurement and computation of the pressure evolution shows differences due to viscous effects. Nevertheless for both, measurement and computation, there is no phase shift between pressure response and shock movement found.

\section{ACKNOWLEDGEMENTS}

The present investigation was carried out as part of a research project funded by ASEA Brown Boveri, Baden, Switzerland (under the supervision of Dr. K. Vogeler) with financial support of the Swiss government. This support and the authorization to publish the results is kindly acknowledged.

\section{REFERENCES}

Adamson, T.C.; Liou, M.S.; 1977

"Unsteady Motion of Shock Waves in Two Dimensional Transonic Channel Flows"

Report No. UM-014534-F, June 1977, Department of Aerospace Engineering, University of Michigan, Michigan, USA

Adamson, T.C.; Liou, M.S.; 1978

"Unsteady Motion of Shock Waves in Two Dimensional Transonic Channel Flows"

Report No. UM-015411-F, October 1978, Department of Aerospace Engineering, University of Michigan, Michigan, USA

Anderson, W.K.; Thomas, J.L.; Rumsey, C.L.; 1985

"Extension of Applications of Flux-Vector Splitting to Unsteady Calculations on Dynamic Meshes"

AIAA Paper 85-0122

Anderson, W.K.; Thomas, J.L.; van Leer, B.; 1987

"A Comparison of Finite Volume Flow With Shock Waves"

AIAA Paper 87-1152

Araki T.; Okamoto Y.; Ohtomo F.; Arinobu M.; 1981

"Self-excited flow oscillation in the low pressure steam turbine cascade"

Communication de L'ITA/ EPFL, No. 10, pp. 171186,1981
Bölcs, A.; Fransson, T.H.; Körbächer, H.; 1991

"Time-dependent pressure fluctuations on an oscillating turbine cascade at transonic off-design flow conditions"

Proceedings of the 6th International Conference on Aeroelasticity in Turbomachines, September 10-15, Notre Dame, Indiana, USA

Bölcs, A.; Fransson, T.H.; Platzer, M.F.; 1989 a

"Numerical Simulation of Inviscid Transonic Flow through Nozzles with Fluctuating Back Pressure"

Journal of Turbomachinery, April 1989, Vol. 111, pp.169-190

Bölcs, A.; Fransson, T. H.; Schläfli, D.; 1989b "Time-Dependent Measurements on Vibrating Annular Turbine Cascades Under Various Steady-State Conditions"

AGARD Conference Proceedings No. 468, "Unsteady Aerodynamic Phenomena in Turbomachines", pp. 19.1-19.14, Luxembourg, August 28-30, 1989

Buffum, D. H.; Fleeter, S.; 1989

"Experimental Investigation of Transonic Oscillating Cascade Aerodynamics"

AIAA paper 89-0321, AIAA 27th Aerospace Sciences Meeting, January 9-12, Reno, Nevada, 1989

Buffum, D. H.; Fleeter, S.; 1990

"Oscillating Cascade Aerodynamics by an Experimental Influence Coefficient Technique"

Journal of Propulsion, Vol. 6, No. 5, pp. 612-620

Buffum, D. H.; Fleeter, S.; 1991

"Wind Tunnel Wall Effects in a Linear Oscillating Cascade"

ASME Paper 91-GT-133, 1991

Carstens, V.; 1991

"Transonic Unsteady Aerodynamics and Aeroelasticity"

73rd Meeting of the AGARD Structures and Materials

Panel, San Diego, California, 7th-11th October, 1991

Edwards, J.A.; 1987

"Surface Pressure Distributions in an Unsteady Shock/Boundary Layer Interaction"

Wolfson College, Cambridge, GB, June 1987

Ezzat, A.; Fransson, T. H.; Jolles, F.; 1989

"Self started Blade Vibrations in an Annular Turbine Cascade Operating at Transonic and Supersonic Mach Numbers"

ASME / IGTI -Publication, Vol. 4 (Editors: Serovy, G. K.; Fransson, T. H.; Fabri, J.)

Fransson, T.H.; 1992

"Analysis of experimental time-dependent blade surface pressures from an oscillating turbine cascade using the influence-coefficient technique"

Journal of Physics III, France , April 1992, pp. 575-594 


\section{Gerolymos, G.A.; 1992a}

"Advances in the Numerical Integration of the 3-D Euler Equations in Vibrating Cascades"

ASME Paper 92-GT-170

Gerolymos, G.A.; 1992b

"Coupled 3-D Aeroelastic Stability Analysis of Bladed Disks"

ASME Paper 92-GT-171

Hanamura, Y.; Yamaguchi, K.; 1988

"An Experimental Investigation on the Flutter of the Cascade of Turbomachinery in the Transonic Flow"

Journal of the Faculty of Engineering, The University of Tokyo (B), Vol. XXXIX, No. 3, pp. 311-338, 1988

Inger, G.R.; 1981

"Application of a Shock-Turbulent Boundary-Layer Interaction Theory in Transonic Flowfield Analysis"

in: "Transonic Aerodynamics", Volume 81 , edited by $D$. Nixon, published by AIAA, ISBN 0-915928-65-5

Liang, S.M.; Tsai, C.J.; Ho, C.K.; 1992

"Numerical Investigation of Unsteady Transonic Nozzle Flows"

AIAA Journal, Vol. 30, No. 2, pp. 566-568, 1992

Ott, P.; 1992

"Oszillierender senkrechter Verdichtungsstoß in einer ebenen Düse"

Communication du Laboratoire de Thermique Appliquée et de Turbomachines Nr. 18, EPFLausanne

Ott, P.; Bölcs, A.; Fransson, T.H.; 1992

"Experimental Study of an Oscillating Normal Shock Wave in a Nozzle"

Proceedings of the 11th Symposium on "Measuring Techniques in Transonic and Supersonic Flows in Cascades and Turbomachines", September 14-15, 1992, München, FRG

Széchényi, E.; Cafarelli, I.; Notin, C.; Girault, J. P.; 1984

"A Straight Cascade Wind-Tunnel Study of Fan Blade Flutter in Started Supersonic Flow"

Proceedings of the Third International Symposium on Unsteady Aerodynamics of Turbomachines and Propellers, Cambridge, UK, September 1984, pp. 447458.

\section{Széchényi, E.; 1985}

"Fan Blade-Single Blade Instability or Blade to Blade Coupling ?"

ASME paper 85-GT-216, Houston, March 1985

Usab, W. J.; Verdon, J. M.; 1990

"Advances in the Numerical Analysis of Linearized Unsteady Cascade Flows"

ASME Paper 90-GT-11, 1990. Also Journal of Turbomachinery, Vol. 113, October 1991, pp. 633-643
Verdon, J. M.; 1989

"The Unsteady Aerodynamic Response to Arbitrary Modes of Blade Motion"

Journal of Fluids and Structures, No. 3, pp. 255-274, 1989

Whitehead, D. S.; Newton, S. G.; 1985

"A Finite Element Method for the Solution of TwoDimensional Transonic Flows in Cascades"

International Journal for Numerical Methods in Fluids, Vol. 5, pp. 115-132, 1985

Whitehead, D. S.; 1990

"A Finite Element Solution of Unsteady TwoDimensional Flow in Cascades"

International Journal for Numerical Methods in Fluids, Vol. 10, pp. 10-34, 1990

\section{APPENDIX 1: NOZZLE FORM}

The shape of the nozzle is a modified derivation of that used by Adamson and Liou [1977, 1978]. The height of the nozzle is computed by the following formula:

$$
H(x)=H_{E n t r y}+\frac{1}{1+3 \cdot \varepsilon^{2}} \cdot \varepsilon^{2} \cdot(f(x)-3)
$$

with

$f(x)= \begin{cases}3 & -0.5 \leq x \leq-\frac{1}{6} \\ \frac{27}{13}[3(x-0.5)+2]^{4}-\frac{48}{13}[3(x-0.5)+2]^{3}+3 & -\frac{1}{6} \leq x \leq \frac{1}{6} \\ \frac{18}{13}[3(x-0.5)]^{2} & \frac{1}{6} \leq x \leq \frac{5}{6} \\ \frac{27}{13}[3(x-0.5)-2]^{4}+\frac{48}{13}[3(x-0.5)-2]^{3}+3 & \frac{5}{6} \leq x \leq \frac{7}{6} \\ 3 & \frac{7}{6} \leq x \leq 1.5\end{cases}$

This places the throat of the nozzle at $x=0$.

The value $\varepsilon$ was chosen as follows:

$$
\varepsilon=0.181568259
$$

For the entry height of $80 \mathrm{~mm}$ we have

$$
\mathrm{H}_{\text {entry }}=0.8[-]
$$

The values for $\mathrm{H}$ and for $\mathrm{x}$ then are multiplied by 100 to obtain the coordinates in $\mathrm{mm}$ for the executed nozzle form in the chosen scale. 


\section{APPENDIX 2: EXIT PRESSURES}

The exit pressures found by an iteration with the objective to obtain the same extreme shock positions as measured were $0.68300[-]$ and $0.73028[-]$. The mean exit pressure for the unsteady computations was thus set to $p=0.70664[-]$ and the exit pressure amplitude for steady state to 0.02364 . With the pressure amplitudes measured for increasing excitation frequency a factor was determined using the pressure difference for steady state pressure as reference. The pressure amplitudes for the computations for different excitation frequencies were found by applying this factor to the steady state pressure difference found by the iteration. The pressure amplitudes used during the computations are presented in Table 1.

\begin{tabular}{|c|c|c|}
\hline \multirow{2}{*}{$\begin{array}{c}\text { Frequency } \\
{[\mathrm{Hz}]}\end{array}$} & \multicolumn{2}{|c|}{ Exit pressure amplitudes } \\
\hline & $\begin{array}{l}\text { measurement } \\
\text { [mbar] }\end{array}$ & $\begin{array}{c}\text { computation } \\
{[-]}\end{array}$ \\
\hline 0 & 32.5 & 0.02364 \\
\hline 20 & 31.0 & 0.02255 \\
\hline 40 & 26.5 & 0.01927 \\
\hline 68 & 25.5 & 0.01856 \\
\hline 80 & 24.0 & 0.01745 \\
\hline 100 & 24.0 & 0.01745 \\
\hline 120 & 22.0 & 0.01600 \\
\hline 140 & 22.0 & 0.01600 \\
\hline 160 & 20.5 & 0.01492 \\
\hline 180 & 19.5 & 0.01418 \\
\hline
\end{tabular}

